\title{
Allopurinol Improves Cardiac Dysfunction After Ischemia- Reperfusion via Reduction of Oxidative Stress in Isolated Perfused Rat Hearts
}

\author{
Yoshiharu Kinugasa, MD; Kazuhide Ogino, MD; Yoshiyuki Furuse, MD; \\ Tetsuya Shiomi, MD*; Hiroyuki Tsutsui, MD*; Tetsuya Yamamoto, MD**; \\ Osamu Igawa, MD; Ichiro Hisatome, MD; Chiaki Shigemasa, MD
}

\begin{abstract}
It has been reported that the xanthine oxidase inhibitor, allopurinol, has a protective effect on ischemia-reperfusion injury, but the precise mechanism of its action is still unclear. Therefore, in the present study the mechanisms of the myocardial protection of allopurinol were evaluated in isolated perfused rat hearts. Allopurinol significantly inhibited myocardial xanthine oxidase activity, and improved left ventricular dysfunction after ischemiareperfusion. In addition, the lactate dehydrogenase content in the coronary effluent obtained after reperfusion was significantly decreased. ATP, ADP, AMP and IMP significantly decreased, whereas inosine, hypoxanthine and xanthine significantly increased after ischemia in both the control and allopurinol groups. The concentration of xanthine was significantly decreased after ischemia-reperfusion in the allopurinol group; however, allopurinol did not affect the other purine metabolites. To evaluate the accumulation of oxidative stress, thiobarbituric acid reactive substances (TBARS) production in myocardial tissue was measured and allopurinol significantly decreased TBARS formation after ischemia-reperfusion. Finally, myocardial hydroxyl radicals were directly measured by electron spin resonance spectroscopy with the nitroxide radical 4-hydroxy-2, 2,6,6-tetramethylpiperidine-N-oxyl. Hydroxyl radicals significantly increased immediately after reperfusion, but were significantly decreased in the allopurinol group. In conclusion, allopurinol reduced myocardial injury after ischemia-reperfusion by suppressing oxidative stress, but not by salvage of ATP. These findings may lead to the development of new therapeutic strategies for myocardial ischemia-reperfusion injury. (Circ J 2003; 67: 781-787)
\end{abstract}

Key Words: Ischemia; Myocardial infarction; Oxygen; Reperfusion

I is well known that ischemia-reperfusion injury is mediated by the production of free radicals in tissues such as the heart, lungs, kidneys, intestine, and brain.,2 In recent years, coronary reperfusion therapy has become a common therapeutic strategy for patients with acute myocardial infarction, but fatal arrhythmia and myocardial dysfunction, known as reperfusion injury, frequently occur immediately after coronary reperfusion and sometimes leads to critical complications. Thus, it is of clinical importance to develop novel therapeutic strategies for ischemia-reperfusion injury.

It has been reported that the xanthine oxidase inhibitor, allopurinol, and its active metabolite, oxypurinol, have a protective effect against ischemia-reperfusion injury in various species: dogs ${ }^{3,4}$ rats, ${ }^{5,6}$ rabbits ${ }^{7,8}$ and humans, 910 The reported effects have been a reduction in the size of the infarction, improvement of ventricular function, ${ }^{4,7}$ a lower incidence of arrhythmias ${ }^{5,8}$ and decreased release of myocardial creatine kinase. However, the precise mechanism

(Received March 20, 2003; revised manuscript received June 9, 2003; accepted June 17, 2003)

Department of Cardiovascular Medicine, Faculty of Medicine, Tottori University, Yonago, *Department of Cardiovascular Medicine, Graduate School of Medical Sciences, Kyushu University, Fukuoka, **Department of Internal Medicine, Hyogo College of Medicine, Nishinomiya, Japan

Mailing address: Kazuhide Ogino, $\mathrm{MD}, \mathrm{PhD}$, Department of Cardiovascular Medicine, Faculty of Medicine, Tottori University, 36-1 Nishimachi, Yonago 683-8504, Japan. E-mail: k-ogino@umin.ac.jp of the protective effect of allopurinol in the ischemic myocardium is still unclear. One possibility is inhibition of free radicals. The conversion of hypoxanthine and xanthine via xanthine oxidase is the major potential source of free radicals, which have been proposed as playing an important role in the genesis of ischemia-reperfusion injury, $3,4,11$ However, it is still controversial whether xanthine oxidase inhibitors improve myocardial injury via a reduction of oxidative stress, because direct measurement of myocardial xanthine oxidase-mediated free radical generation has not been performed.

In the present study, therefore, we directly measured myocardial hydroxyl radicals by electron spin resonance (ESR) spectroscopy using for the first time the nitroxide radical 4-hydroxy-2,2,6,6-tetramethyl-piperidine- $\mathrm{N}$-oxyl (hydroxy-TEMPO).

\section{Methods}

\section{Isolated Heart Preparation}

Male Wistar rats (300-350g, 12 weeks old) were used for the isolated isovolumic heart preparations. All animals were handled in strict accordance with the Tottori University Guide for the Care and Use of Laboratory Animals. Isolated perfused rat hearts were created as previously described! ${ }^{2}$ In brief, after the rats were heavily anaesthetized by intraperitoneal injection of a cocktail of ketamine $\mathrm{HCl}$ (40 mg, Sigma, St Louis, MO, USA) and xylazine ( $2.0 \mathrm{mg}$, Sigma) dissolved in heparin (1,000 IU, Shimizu Seiyaku, 
Shimizu, Japan), the heart was rapidly excised, and the aorta was cannulated for retrograde perfusion with a 14gauge needle connected to a modified Langendorff perfusion system, which consisted of a warmed storage vat and a condenser at $37^{\circ} \mathrm{C}$, and an adjustable-speed rotary pump (model 7523-40, Masterflex, Barrington, IL, USA). Throughout the experiment, the coronary perfusion pressure (CPP) was held at $80 \mathrm{mmHg}$ by changing the coronary perfusion flow of modified Tyrode's solution (in mmol/L: $\mathrm{NaCl} 144, \mathrm{KCl} 5, \mathrm{CaCl}_{2} 1.5, \mathrm{MgCl}_{2}$ 0.9, N-[2-hydroxyethyl] piperazine-N'-[2-ethanesulphonic acid] (HEPES) 6, and glucose $5, \mathrm{pH} 7.4$ ) equilibrated with $100 \%$ oxygen. The perfusate was not recirculated. Ventricular function was assessed by measuring the left ventricular pressure (LVP) with a fluid-filled latex balloon inserted into the left ventricle through the mitral valves and inflated to give an end-diastolic pressure of $5 \mathrm{mmHg}$. The transducer was connected to a Mac Lab System (model Power Lab 8sp, AD Instruments, Castle Hill, NSW, Australia) and the LVP and CPP were measured. After being attached to the Langendorff perfusion system, the heart was allowed to stabilize for $30 \mathrm{~min}$, during which time LVP and CPP (constant CPP at $80 \mathrm{mmHg}$ ) were monitored.

\section{Experimental Protocol}

All hearts were perfused for $30 \mathrm{~min}$ to allow stabilization of LVP and CPP, and then subjected to $25 \mathrm{~min}$ of global ischemia, followed by $30 \mathrm{~min}$ of reperfusion. In the allopurinol group, the hearts were perfused with allopurinol ( $1.0 \mathrm{mmol} / \mathrm{L}$, Sigma) dissolved directly in the Tyrode's solution for $10 \mathrm{~min}$ before ischemia, and continuously for $30 \mathrm{~min}$ of reperfusion. During the ischemic period, the hearts were placed in Tyrode's solution in a water-jacketed container to maintain the temperature constant at $37^{\circ} \mathrm{C}$. We measured preischemic and postischemic values for LVP, $\mathrm{CPP}$ and coronary perfusion flow throughout the experiment. To eliminate the effect of changes in heart rate, pacing was performed at a constant rate $(240$ beats $/ \mathrm{min}$ ) before ischemia and after reperfusion using a pacing generator (model SEN3201, Nihon Kohden, Tokyo, Japan) as previously described! ${ }^{3}$ Myocardial tissue was collected immediately before global ischemia, immediately before reperfusion and at 1,5 and $30 \mathrm{~min}$ after reperfusion. The samples were quickly frozen in liquid nitrogen and stored at $-80^{\circ} \mathrm{C}$ until assays for the measurement of purine metabolites and reactive oxygen species (ROS).

\section{Measurement of Lactate Dehydrogenase (LDH) Activity}

To analyze the release of $\mathrm{LDH}$, coronary effluent was also collected for $1 \mathrm{~min}$ before and after reperfusion. $\mathrm{LDH}$ activity was determined using an LDH assay kit (cicaliquid LDH, Kantokagaku, Tokyo, Japan). The absorbance was measured at $340 \mathrm{~nm}$. LDH activity was expressed as IU. $\min ^{-1} \cdot \mathrm{g}^{-1}$ wet tissue weight.

\section{Measurement of Xanthine Oxidase Activity}

Xanthine oxidase activity was measured as previously described ${ }^{14,15}$ Briefly, fresh myocardial tissue was homogenized in ice-cold $5 \mathrm{mmol} / \mathrm{L}$ phosphate buffer $(\mathrm{pH} 7.4)$ containing $1.0 \mathrm{mmol} / \mathrm{L}$ EDTA, $1.0 \mathrm{mmol} / \mathrm{L}$ dithiothreitol and $0.1 \mathrm{mmol} / \mathrm{L}$ phenyl-methyl sulfonyl fluoride. The mixture was centrifuged at $7,700 \mathrm{G}$ for $30 \mathrm{~min}$ at $4^{\circ} \mathrm{C}$ (Model RM150, Tomyseiko, Tokyo, Japan). After preincubation for $5 \mathrm{~min}$ at $37^{\circ} \mathrm{C}$ of $240 \mu 10.2 \mathrm{~mol} / \mathrm{L}$ Tris- $\mathrm{HCl}$ buffer $(\mathrm{pH} 9.0)$ containing $113 \mu \mathrm{mol} / \mathrm{L}$ pterin with or without $4 \mathrm{mmol} / \mathrm{L}$
NAD, the enzyme reaction was started by adding $60 \mu 1$ of the supernatant. After incubation for 10 to $30 \mathrm{~min}, 100 \mathrm{\mu} \mathrm{l}$ of the reaction mixture were removed and added to $1.5-\mathrm{ml}$ tubes containing $100 \mu \mathrm{l}$ of $4 \% \mathrm{HClO}_{4}$. The resulting mixture was vigorously shaken with an agitator and then centrifuged at $15,000 \mathrm{G}$. A portion $(150 \mu \mathrm{l})$ of the supernatant was neutralized with $6 \mu 1$ of $5 \mathrm{~mol} / \mathrm{L} \mathrm{K}_{2} \mathrm{CO}_{3}$, and $20 \mu \mathrm{l}$ of the neutralized supernatant were used to measure xanthine oxidase activity by high-performance liquid chromatography (HPLC) (LC-6A HPLC apparatus, RF 530 fluorescence HPLC monitor, C-R3A chromatopac recorder, Shimadzu, Kyoto, Japan). Xanthine oxidase activity was expressed as $\mathrm{nmol} \cdot \mathrm{g}$ protein ${ }^{-1} \cdot \mathrm{h}^{-1}$.

\section{Measurement of Adenine Nucleotides and Purine \\ Metabolites in Myocardial Tissue}

Adenine nucleotides and purine metabolites were measured as previously described ${ }^{16}$ Frozen myocardial tissue was homogenized $(10 \% \mathrm{wt} / \mathrm{vol})$ in $6 \%$ trichloroacetic acid (TCA) and centrifuged at $17,000 \mathrm{G}$ for $10 \mathrm{~min}$ at $4^{\circ} \mathrm{C}(\mathrm{MX}-$ 180 centrifuge, Tomyseiko, Tokyo, Japan). The supernatant was then removed and added to an equal volume of the tri- $n$-octylamine in freon (Sigma), vortex-mixed for $2 \mathrm{~min}$, and centrifuged at $700 \mathrm{G}$ for $3 \mathrm{~min}$. The aqueous layer was filtered through a $0.22 \mu \mathrm{m}$ Millipore filter. The amount of purine in myocardial tissue was determined by HPLC (L6000 pump, L4000 UV Detector, HITACHI, Tokyo, Japan). To separate nucleotides [adenosine triphosphate (ATP), adenosine diphosphate (ADP), adenosine monophosphate (AMP), inosine monophosphate (IMP)], we used a mobile phase buffer containing $10 \mathrm{mmol} / \mathrm{L} \mathrm{NaH}_{2} \mathrm{PO}_{4}$, $5 \mathrm{mmol} / \mathrm{L}$ tetrabutyl-ammonium-bromide, and acetonitrile, adjusted to $\mathrm{pH}$ 6.0. Nucleotides (inosine, hypoxanthine, xanthine) were then separated using the buffer, $60 \mathrm{nmol} / \mathrm{L}$ $\mathrm{KH}_{2} \mathrm{PO}_{4}, 0.45 \mathrm{mmol} / \mathrm{L}$ tetra-n-butylammonium phosphate and $0.35 \mathrm{~mol} / \mathrm{L}$ acetonitrile, adjusted to $\mathrm{pH} 3.0$. All buffers were filtered through $0.45 \mu \mathrm{m}$ Millipore filters. Nucleotides and nucleosides were identified by comparing their retention time with those of standard samples [ATP, ADP, AMP, IMP, inosine, hypoxanthine, xanthine (Sigma)], and we determined the concentration of each sample on the basis of the peak area using external standards. The amount of purine metabolites in myocardium was expressed as $\mu \mathrm{mol} / \mathrm{g}$ wet tissue weight.

\section{Measurement of Thiobarbituric Acid Reactive Substances (TBARS)}

Myocardial tissue was homogenized $(10 \% \mathrm{wt} / \mathrm{vol})$ in $1.15 \%$ KCL solution $(\mathrm{pH} 7.4)$. The homogenate $(0.1 \mathrm{ml})$ was mixed with $50 \mu \mathrm{1}, 0.8 \%$ butylated hydroxytoluene solution in glacial acetic acid, $0.20 \mathrm{ml}$ of $8.1 \%$ sodium dodecyl sulfate (SDS) solution, $1.50 \mathrm{ml}$ of $20 \%$ acetic acid solution adjusted at $\mathrm{pH} 3.5$ with $10 \mathrm{~mol} / \mathrm{L} \mathrm{NaOH}, 0.70 \mathrm{ml}$ water and $1.50 \mathrm{ml}$ of $0.8 \%$ thiobarbituric acid solution. The mixture was kept at $5^{\circ} \mathrm{C}$ for $60 \mathrm{~min}$ and then heated at $100^{\circ} \mathrm{C}$ for $60 \mathrm{~min}$. After cooling, the mixture was extracted with $1.0 \mathrm{ml}$ water and $5.0 \mathrm{ml} \mathrm{n}$-butanol:pyridine $(15: 1 \mathrm{v} / \mathrm{v})$ and centrifuged at $700 \mathrm{G}$ for $10 \mathrm{~min}$. The absorbance of the organic phase at $532 \mathrm{~nm}$ was measured (220 A spectrophotometer, HITACHI, Tokyo, Japan). The amount of TBARS was determined by the absorbance with the molecular extinction coefficient of 156,000 and expressed as $\mathrm{nmol} / \mathrm{g}$ wet tissue weight ${ }^{17,18}$ 
Table 1 Effect of Allopurinol on Left Ventricular Function Before and After Ischemia-Reperfusion

\begin{tabular}{lccccc}
\hline \hline & \multicolumn{2}{c}{ Before ischemia } & & \multicolumn{2}{c}{ 30 min after reperfusion } \\
\cline { 2 - 3 } \cline { 5 - 6 } & Control $(n=5)$ & Allopurinol $(n=5)$ & & Control $(n=5)$ & Allopurinol $(n=5)$ \\
\hline LVPmax $(\mathrm{mmHg})$ & $107.0 \pm 1.7$ & $106.2 \pm 3.2$ & & $96.1 \pm 2.9^{*}$ & $110.5^{*} \pm 5.3^{\dagger}$ \\
LVEDP $(\mathrm{mmHg})$ & $5.0 \pm 0.1$ & $5.1 \pm 0.1$ & & $39.1 \pm 6.0^{*}$ & $23.1 \pm 3.2^{* \dagger}$ \\
Developed pressure $(\mathrm{mmHg})$ & $102.0 \pm 1.7$ & $101.1 \pm 3.3$ & & $57.0 \pm 5.8^{*}$ & $87.4 \pm 5.7^{*+}$ \\
dP/dtmax $(\mathrm{mmHg} / \mathrm{s})$ & $2,982 \pm 63$ & $2,936 \pm 78$ & & $1,284 \pm 156^{*}$ & $1,949 \pm 130^{*+}$ \\
dP/dtmin $(\mathrm{mmHg} / \mathrm{s})$ & $-1,903 \pm 59$ & $-1,766 \pm 69$ & & $-848 \pm 78^{*}$ & $-1,353 \pm 99^{* \dagger}$ \\
\hline
\end{tabular}

LVPmax, peak left ventricular pressure; LVEDP, left ventricular end-diastolic pressure; dP/dtmax, peak rate of rise of LVP; $d P / d t m i n$. peak rate of fall of $L V P$.

${ }^{*} p<0.05$ vs before ischemia, ${ }^{t} p<0.05$ vs control.

\section{Measurement of ROS in Myocardial Tissue}

ROS were quantified in tissues by ESR spectroscopy with hydroxy-TEMPO as previously described 19 In brief, myocardial tissue was homogenized in $50 \mathrm{mmol} / \mathrm{L}$ sodium phosphate buffer ( $\mathrm{pH}$ 7.4) containing protease inhibitors. First, the homogenate was immediately reacted with hydroxy-TEMPO $(0.1 \mathrm{mmol})$, and its ESR spectra were recorded using an X-band $(9.45-\mathrm{GHz})$ ESR spectrometer (JES-FA100/BU, JEOL, Tokyo, Japan). Second, dimethylthiourea (DMTU; $50 \mathrm{mmol} / \mathrm{L}$ ), a specific $\mathrm{OH}^{-}$scavenger, was also added to the reaction mixture and we measured the decrease in signal decay after the addition of DMTU, which indicates the amount of hydroxyl radicals.

\section{Statistical Analysis}

Comparisons among multiple groups were determined by two-way analysis of variance with Bonferroni's post hoc test. Comparisons for values between the control and allopurinol groups were performed by unpaired Student's t-test as appropriate. All data are expressed as the mean value \pm SEM; $p<0.05$ was considered statistically significant.

\section{Results}

\section{Effect of Allopurinol on LVP}

LVP, peak LVP (LVPmax), left ventricular end-diastolic pressure (LVEDP), developed pressure, peak rate of rise of LVP (dP/dtmax) and peak rate of fall of LVP (dP/dtmin) before global ischemia and after reperfusion in the control and allopurinol groups are summarized in Table 1. There were no significant differences in LVPmax, LVEDP, developed pressure, $\mathrm{dP} / \mathrm{dtmax}$ or $\mathrm{dP} / \mathrm{dtmin}$ before ischemia between the control and allopurinol groups, suggesting that allopurinol had no significant effect on left ventricular function before ischemia. After ischemia, LVPmax, developed pressure, $\mathrm{dP} / \mathrm{dtmax}$ and $\mathrm{dP} / \mathrm{dtmin}$ were significantly decreased and LVEDP was significantly increased in both the control and allopurinol group; however, in hearts treated with allopurinol, the changes in LVPmax, developed pressure, $\mathrm{dP} / \mathrm{dtmax}$ and $\mathrm{dP} / \mathrm{dtmin}$ and LVEDP were significantly attenuated in comparison with those of control hearts $(p<0.05)$. These data suggest that allopurinol significantly improved left ventricular function after ischemiareperfusion.

\section{Effect of Allopurinol on LDH Activity}

We measured LDH activity in the coronary effluent as an index of myocardial cellular injury after ischemiareperfusion. Fig 1 shows the time course of LDH in the coronary effluent before and after ischemia-reperfusion. LDH activity significantly increased after reperfusion and

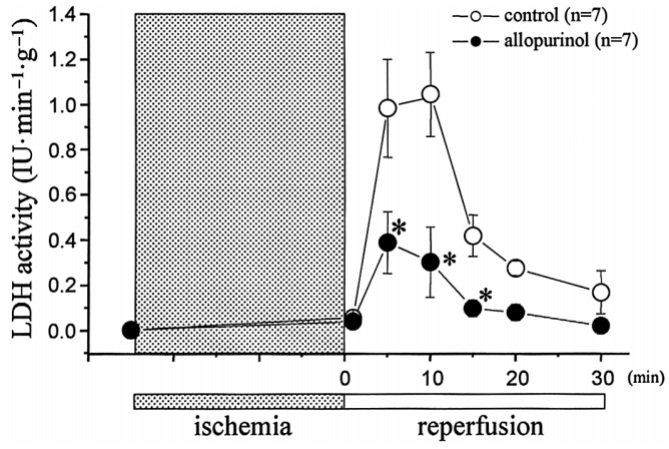

Fig 1. Effect of allopurinol on lactate dehydrogenase activity before ischemia and after reperfusion. ${ }^{*} \mathrm{p}<0.05$ vs control.

peaked between 5 and $10 \mathrm{~min}$ after reperfusion in both groups. In the allopurinol group, LDH activity was significantly lower at $5 \mathrm{~min}, 10 \mathrm{~min}$ and $15 \mathrm{~min}$ after reperfusion compared with the control group ( $5 \mathrm{~min}: 0.39 \pm 0.14$ and $0.98 \pm 0.22,10$ min: $0.30 \pm 0.16$ and $1.04 \pm 0.19,15$ min: $0.10 \pm$ 0.04 and $0.42 \pm 0.09 \mathrm{IU} \cdot \mathrm{min}^{-1} \cdot \mathrm{g}^{-1}$, respectively, $\mathrm{p}<0.05$ ). Therefore, allopurinol significantly decreased the release of LDH from the myocardium, suggesting attenuation of the myocardial damage caused by ischemia.

\section{Effect of Allopurinol on Adenine Nucleotides, and Purine Metabolites in Myocardium}

Fig 2 shows the time course of the ATP, ADP, AMP, IMP, inosine, hypoxanthine and xanthine concentrations in myocardial tissue before and after ischemia-reperfusion. There were no significant differences in these concentrations before global ischemia between the control and allopurinol groups. The concentrations of ATP, ADP, AMP and IMP significantly decreased after reperfusion in both the control and allopurinol groups, with no differences between the 2 groups (Fig 2A-D). The concentrations of inosine, hypoxanthine and xanthine significantly increased after reperfusion and gradually decreased during reperfusion (Fig 2E-G). The concentration of xanthine in the allopurinol group was significantly lower after reperfusion compared with the control (0 min: $0.191 \pm 0.041$ and $0.076 \pm 0.014,1 \mathrm{~min}: 0.103 \pm 0.012$ and $0.051 \pm 0.010,5 \mathrm{~min}$ : $0.075 \pm 0.017$ and $0.025 \pm 0.007 \mu \mathrm{mol} / \mathrm{g}$, respectively, $\mathrm{p}<0.05$ ) whereas there were no significant differences in the concentration of inosine or hypoxanthine between the 2 groups. Taken together, these results suggest that allopurinol does not preserve myocardial ATP; however, it reduces the myocardial concentration of xanthine. 
A

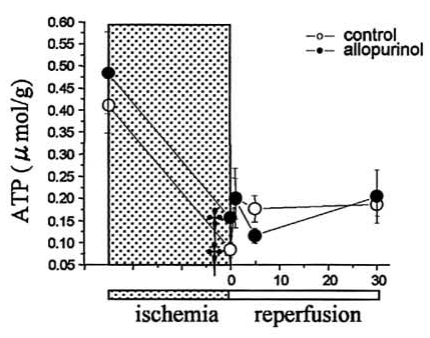

C

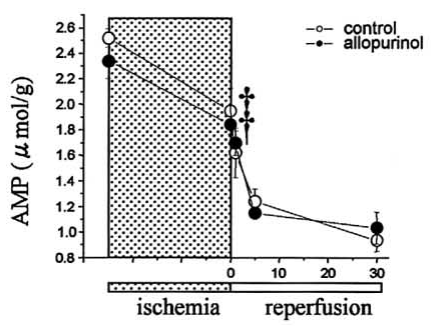

E
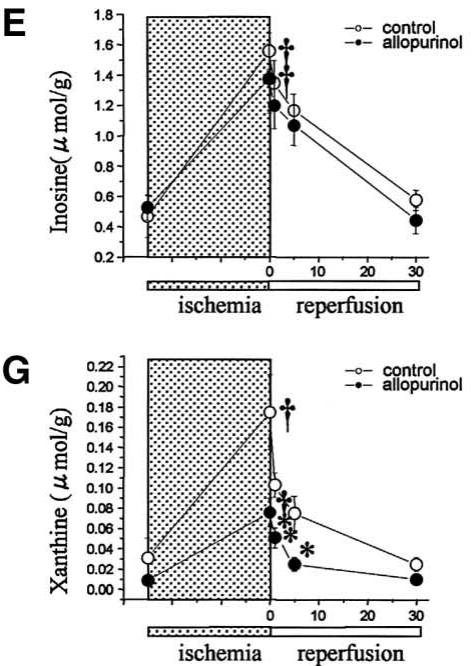

B

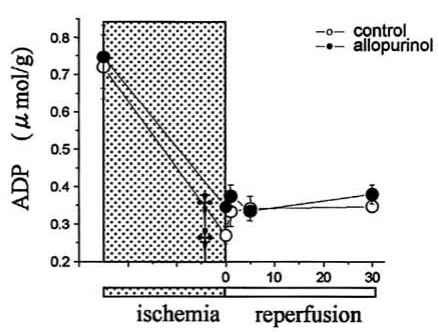

D

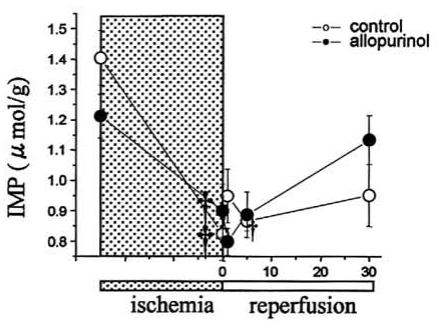

\section{$\mathbf{F}$}

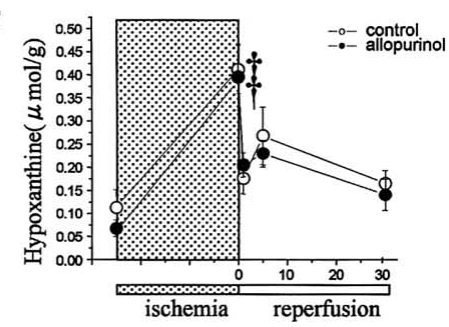

Fig 2. Time course of the concentrations of ATP, ADP, AMP, IMP, inosine, hypoxanthine and xanthine during ischemia and reperfusion. ${ }^{*} \mathrm{p}<0.05$ vs control, ${ }^{\dagger} \mathrm{p}<0.05$ vs pre-ischemia; $\mathrm{n}=5-7$ at each point.

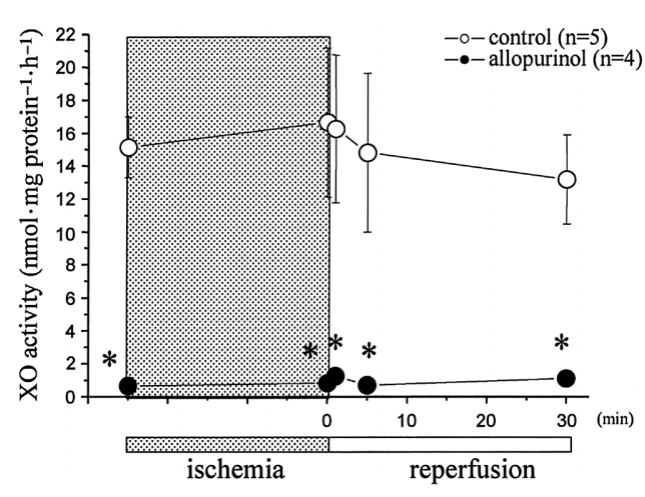

Fig 3. Effect of allopurinol on xanthine oxidase $(\mathrm{XO})$ activity during ischemia and reperfusion. ${ }^{*} \mathrm{p}<0.05$ vs control.

\section{Effect of Allopurinol on Xanthine Oxidase Activity During Ischemia-Reperfusion}

In order to determine whether or not xanthine oxidase activity is increased after ischemia and reperfusion and is suppressed by allopurinol, we measured the enzyme activity in the myocardium before and after ischemia-

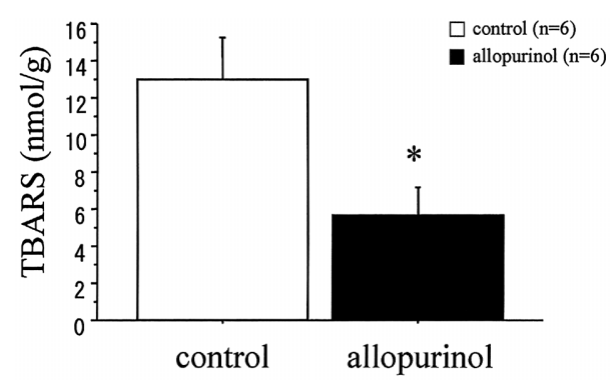

Fig 4. Effect of allopurinol on the production of TBARS in myocardial tissue $30 \mathrm{~min}$ after ischemia -reperfusion. ${ }^{*} \mathrm{p}<0.05$ vs control.

reperfusion. Fig 3 shows the time course of xanthine oxidase activity before and after ischemia-reperfusion. Xanthine oxidase activity did not change significantly after ischemia-reperfusion; however, the enzyme activity in hearts treated with allopurinol was significantly decreased compared with the control hearts before and after ischemia-reperfusion. Thus, the xanthine oxidase activity did not change after ischemia-reperfusion, but it was significantly inhibited by allopurinol. 


\section{Effect of Allopurinol on the Production of TBARS After Ischemia-Reperfusion}

Lipid peroxidation products were measured as a marker of accumulation of oxidative injury. Fig 4 shows the production of TBARS after reperfusion in control and allopurinol-treated hearts. TBARS production was significantly lower in hearts treated with allopurinol than in control hearts $(5.6 \pm 1.5$ and $13.0 \pm 2.3 \mathrm{nmol} / \mathrm{g}$, respectively, $\mathrm{p}<0.05)$.

\section{Effect of Allopurinol on Hydroxyl Radicals Generation After Ischemia-Reperfusion}

Fig 5 shows the time course of ESR signal decay of hydroxy-TEMPO just before and after reperfusion in the control and allopurinol groups. There were no significant differences in ESR signal decay of hydroxy-TEMPO just before reperfusion between the control and allopurinol groups. In the control group, the ESR signal decay of hydroxy-TEMPO was significantly increased at $1 \mathrm{~min}$ after reperfusion and then gradually decreased throughout reperfusion. On the other hand, allopurinol significantly suppressed the increased signal decay at $1 \mathrm{~min}$ and $5 \mathrm{~min}$ compared with the control group ( 1 min: $0.028 \pm 0.003$ and $0.019 \pm 0.002,5 \mathrm{~min}: 0.018 \pm 0.002$ and $0.007 \pm 0.003$, respectively, $\mathrm{p}<0.05)$. These data suggest that allopurinol significantly reduces the generation of hydroxyl radicals after ischemia-reperfusion.

\section{Discussion}

The results of the present study show that myocardial hydroxyl radicals significantly increased after ischemiareperfusion and significantly decreased in allopurinoltreated animals; moreover this decrease was accompanied by improved cardiac function after ischemia-reperfusion. In addition, the production of TBARS was also suppressed by allopurinol. Furthermore, allopurinol significantly inhibited myocardial xanthine oxidase activity and decreased the myocardial concentration of xanthine after ischemiareperfusion.

The present findings suggest that allopurinol prevents the myocardial dysfunction caused by ischemia-reperfusion injury by suppressing ROS. It has been proposed that xanthine oxidase plays an important role in the generation of free radicals after ischemia-reperfusion, $3,4,11,20,21$ and that xanthine oxidase inhibitors have protective effects against ischemia-reperfusion injury?-10 However, it is still unclear whether the reduction of free radicals is involved in the beneficial effect of allopurinol after ischemia-reperfusion. Using ESR spectroscopy with 5,5-dimethyl-1-pyroline-N-oxide, Thompson-Gorman et al demonstrated that oxypurinol significantly decreased ROS in the coronary effluent after ischemia-reperfusion ${ }^{22}$ On the other hand, Pisarenko et al failed to demonstrate the decrease of ROS in the coronary effluent with allopurinol using the same method ${ }^{23}$ In those studies, myocardial free radicals were not directly measured. Thus, ours is the first report to demonstrate myocardial ROS generation after ischemia-reperfusion using ESR with hydroxy-TEMPO.

ATP, ADP, AMP and IMP significantly decreased, whereas inosine, hypoxanthine and xanthine significantly increased after ischemia, which means that ATP degradation occurs in the hearts during ischemia followed by accumulation of the purine metabolites that are sources of ROS generation (ie, inosine, hypoxanthine and xanthine).

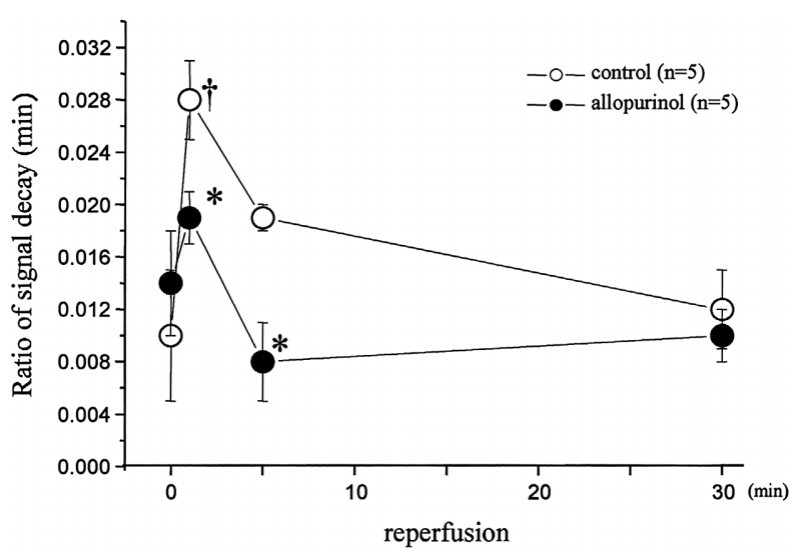

Fig 5. Effect of allopurinol on hydroxyl radical generation after ischemia-reperfusion. ${ }^{*} \mathrm{p}<0.05$ vs control, ${ }^{\dagger} \mathrm{p}<0.05$ vs $0 \mathrm{~min}$.

However, our results showed that allopurinol did not retain ATP and did not affect the concentrations of these purine metabolites, except for that of xanthine, after ischemiareperfusion, which suggests that the beneficial effects of allopurinol on myocardial dysfunction after ischemiareperfusion are not mediated by the salvage of adenine nucleotides, consistent with the results of a previous study 22 On the other hand, it has been reported that ATP is decreased and AMP increased during ischemia, 24,25 and that xanthine oxidase inhibitors can improve post-ischemic left ventricular function via the salvage and preservation of adenine nucleotides ${ }^{23,26,27}$ These discrepant results might be attributed to different experimental protocols. In our study long-term ischemia may have led to a rapid degradation of purine metabolites followed by a decrease in AMP, whereas the perfusion of allopurinol throughout the entire experiment or the combination with long-term pretreatment prior to heart excision may have increased the efficiency of ATP salvage in the previous study ${ }^{23}$ compared with the acute administration of the drug prior to and after ischemia in the present study. The shorter duration of ischemia (10-15 min) in previous studies may also account for the increased efficiency of ATP salvage ${ }^{26,27}$ Further studies are needed to clarify the involvement of energy preservation in the protective effect of allopurinol on myocardial dysfunction after ischemia-reperfusion.

The increase in the activity of xanthine oxidase after ischemia-reperfusion has been proposed as a key role in the generation of free radicals 11,28 It has been suggested that xanthine dehydrogenase is converted to xanthine oxidase during ischemia ${ }^{29}$ and xanthine oxidase activity has been found to increase relative to dehydrogenase during ischemia?22 However, the activity of xanthine oxidase did not significantly change throughout the entire experiment in the present study, whereas it was completely inhibited by allopurinol. In addition, we confirmed using Western blot that the amount of myocardial xanthine oxidase was not significantly affected by allopurinol before or after ischemia-reperfusion (data not shown). No significant conversion of xanthine dehydrogenase to xanthine oxidase has been previously observed in ischemic rat hearts ${ }^{30,31}$ and the alteration in xanthine oxidase activity is not the major limiting factor in the process of free radicals generation during postischemic reperfusion. ${ }^{1}$ In the present study, myocardial substrate (ie, inosine, hypoxanthine and xanthine) levels rapidly decreased after reperfusion, proba- 
bly because of both substrate metabolism and washout, followed by decreases in hydroxyl radicals (Fig 5). These results indicate that an increase in the substrate of xanthine oxidase, rather than an increased xanthine oxidase activity or the amount of xanthine oxidase, is responsible for the generation of free radicals after ischemia-reperfusion. Further studies will be needed to clarify which mechanism is more important for the decrease in ROS associated with allopurinol treatment.

Although the level of xanthine oxidase activity is significant in the myocardium of rats and dogs, it is relatively low in the myocardium of rabbits and humans ${ }^{32,33}$ which makes it unclear whether the same principle of xanthine oxidase inhibition can be applied to human myocardial protection against ischemia-reperfusion injury. Some reports deny the protective effect of allopurinol on ischemia-reperfusion injury in rabbit hearts; $; 4,35$ however, Terada et al reported that allopurinol improved left ventricular function in rabbit hearts? In addition, pretreatment with allopurinol has been shown to improve postoperative recovery 9,10 and reduce lipid peroxidation ${ }^{9}$ in patients undergoing open-heart surgery; those reports suggest that allopurinol may contribute to myocardial protection after ischemia-reperfusion in humans. Immunochemistry of human tissue has revealed that xanthine oxidase is mainly localized in the vascular endothelium and smooth muscle cells 36,37 Thus, xanthine oxidase from coronary endothelial/smooth muscle cells may contribute to the production of free radicals after ischemia-reperfusion. On the other hand, Saugstad postulated that xanthine oxidase originates from other organs, such as the liver and intestines, reaches the heart and produces free radicals under hypoxic conditions ${ }^{33}$ In addition, it has been reported that circulating plasma xanthine oxidase contributes to vascular endothelium dysfunction 38 Taking those findings together, it is possible that xanthine oxidase originating from other organs contributes to the generation of free radicals in the heart after ischemia reperfusion; however, it is unclear to what extent circulating xanthine oxidase is important for ischemia-reperfusion injury. Further studies are necessary to clarify the exact mechanism of myocardial ischemia-reperfusion injury and the protection of the myocardium by allopurinol in various species.

In conclusion, allopurinol attenuated myocardial dysfunction in the rat heart after ischemia-reperfusion by suppressing ROS generation, not by the salvage of ATP. This is the first report to directly measure myocardial ROS and thus demonstrate the clinical importance of reducing them ischemia-reperfusion injury. Allopurinol has a well-established safety profile and is used widely for the treatment of gout. It has, therefore, the potential to be developed as a novel therapeutic strategy for ischemia-reperfusion injury.

\section{References}

1. Midorikawa J, Maehara K, Yaoita H, Watanabe T, Ohtani H, Ushiroda S, et al. Continuous observation of superoxide generation in an in-situ ischemia-reperfusion rat lung model. Jpn Circ J 2001; 65: $207-212$.

2. Kaneda T, Ku K, Inoue T, Onoe M, Oku H. Postischemic reperfusion injury can be attenuated by oxygen tension control. Jpn Circ J 2001; 65: $213-218$.

3. Chambers DE, Parks DA, Patterson G, Roy R, McCord JM, Yoshida $\mathrm{S}$, et al. Xanthine oxidase as a source of free radical damage in myocardial ischemia. J Mol Cell Cardiol 1985; 17: 145-152.

4. Stewart JR, Crute SL, Loughlin V, Hess ML, Greenfield LJ. Prevention of free radical-induced myocardial reperfusion injury with allopurinol. J Thorac Cardiovasc Surg 1985; 90: 68-72.
5. Manning AS, Coltart DJ, Hearse DJ. Ischemia and reperfusioninduced arrhythmias in the rat: Effects of xanthine oxidase inhibition with allopurinol. Circ Res 1984; 55: 545-548.

6. Miwa-Nishimura N, Kanaide H, Abe S, Nakamura M. Does allopurinol prevent myocardial injury as a result of hypoxia-re-oxygenation in rats? Int J Exp Pathol 1990; 71: 727-739.

7. Terada LS, Rubinstein JD, Lesnefsky EJ, Horwitz LD, Leff JA, Repine JE. Existence and participation of xanthine oxidase in reperfusion injury of ischemic rabbit myocardium. Am J Physiol 1991; 260: $\mathrm{H} 805-\mathrm{H} 810$

8. Godin DV, Bhimji S, McNeill JH. Effects of allopurinol pretreatment on myocardial ultrastructure and arrhythmias following coronary artery occlusion and reperfusion. Virchows Arch B Cell Pathol Incl Mol Pathol 1986; 52: 327-341.

9. Coghlan JG, Flitter WD, Clutton SM, Panda R, Daly R, Wright G, et al. Allopurinol pretreatment improves postoperative recovery and reduces lipid peroxidation in patients undergoing coronary artery bypass grafting. J Thorac Cardiovasc Surg 1994; 107: 248-256.

10. Adachi H, Motomatsu K, Yara I. Effect of allopurinol (zyloric) on patients undergoing open heart surgery. Jpn Circ J 1979; 43: 395401.

11. McCord JM. Oxygen-derived free radicals in postischemic tissue injury. N Engl J Med 1985; 312: 159-163.

12. Furuse Y, Ogino K, Shimoyama M, Sasaki N, Hisatome I. Ca ${ }^{2+}$-sensitizing effect is involved in the positive inotropic effect of troglitazone. Br J Pharmacol 2001; 133: 1307-1313.

13. Shimoyama M, Ogino K, Tanaka Y, Ikeda T, Hisatome I. Hemodynamic basis for the acute cardiac effects of troglitazone in isolated perfused rat hearts. Diabetes 1999; 48: 609-615.

14. Moriwaki Y, Yamamoto T, Suda M, Nasako Y, Takahashi S, Agbedana $\mathrm{O}$, et al. Purification and immunohistochemical tissue localization of human xanthine oxidase. Biochim Biophys Acta 1993; 1164: $327-330$.

15. Yamamoto T, Moriwaki Y, Takahashi S, Tsutsumi Z, Yamakita J, Nasako Y, et al. Determination of human plasma xanthine oxidase activity by high-performance liquid chromatography. J Chromatogr B Biomed Appl 1996; 681: 395-400.

16. Tekkanat KK, Fox IH. Isocratic separation of ATP and its degradation products from biological fluids by automated liquid chromatography. Clin Chem 1988; 34: 925-932.

17. Kikugawa K, Kojima T, Yamaki S, Kosugi H. Interpretation of the thiobarbituric acid reactivity of rat liver and brain homogenates in the presence of ferric ion and ethylenediaminetetraacetic acid. Anal Biochem 1992; 202: 249-255.

18. Ide T, Tsutsui H, Kinugawa S, Utsumi H, Kang D, Hattori N, et al. Mitochondrial electron transport complex I is a potential source of oxygen free radicals in the failing myocardium. Circ Res 1999; 85: $357-363$.

19. Ide T, Tsutsui H, Kinugawa S, Suematsu N, Hayashidani S, Ichikawa $\mathrm{K}$, et al. Direct evidence for increased hydroxyl radicals originating from superoxide in the failing myocardium. Circ Res 2000; 86: $152-$ 157.

20. Kuppusamy P, Zweier JL. Characterization of free radical generation by xanthine oxidase: Evidence for hydroxyl radical generation. $J$ Biol Chem 1989; 264: 9880-9884.

21. Xia Y, Zweier JL. Substrate control of free radical generation from xanthine oxidase in the postischemic heart. J Biol Chem 1995; 270: $18797-18803$

22. Thompson-Gorman SL, Zweier JL. Evaluation of the role of xanthine oxidase in myocardial reperfusion injury. J Biol Chem 1990; 265: 6656-6663.

23. Pisarenko OI, Lakomkin VL, Studneva IM, Timoshin AA, Kuzmin AI, Ruuge EK, et al. Allopurinol-enhanced postischemic recovery in the isolated rat heart involves repletion of high-energy phosphates. Biochem Med Metab Biol 1994; 51: 16-26.

24. Harrison GJ, Willis RJ, Headrick JP. Extracellular adenosine levels and cellular energy metabolism in ischemically preconditioned rat heart. Cardiovasc Res 1998; 40: 74-87.

25. Angelos MG, Murray HN, Gorsline RT, Klawitter PF. Glucose, insulin and potassium (GIK) during reperfusion mediates improved myocardial bioenergetics. Resuscitation 2002; 55: 329-336.

26. Lasley RD, Ely SW, Berne RM, Mentzer RM Jr. Allopurinol enhanced adenine nucleotide repletion after myocardial ischemia in the isolated rat heart. J Clin Invest 1988; 81: 16-20.

27. Khatib SY, Farah H, El-Migdadi F. Allopurinol enhances adenine nucleotide levels and improves myocardial function in isolated hypoxic rat heart. Biochemistry (Mosc) 2001; 66: 328-333.

28. Hearse DJ, Manning AS, Downey JM, Yellon DM. Xanthine oxidase: A critical mediator of myocardial injury during ischemia and reperfusion? Acta Physiol Scand Suppl 1986; 548: 65-78. 
29. McCord JM, Roy RS. The pathophysiology of superoxide: Roles in inflammation and ischemia. Can J Physiol Pharmacol 1982; 60: $1346-1352$

30. Kehrer JP, Piper HM, Sies H. Xanthine oxidase is not responsible for reoxygenation injury in isolated-perfused rat heart. Free Radic Res Commun 1987; 3: 69-78.

31. Chambers DJ, Takahashi A, Humphrey SM, Harvey DM, Hearse DJ. Allopurinol-enhanced myocardial protection does not involve xanthine oxidase inhibition or purine salvage. Basic Res Cardiol 1992; 87: 227-238.

32. McCord JM. Free radicals and myocardial ischemia: Overview and outlook. Free Radic Biol Med 1988; 4: 9-14.

33. Saugstad OD. Role of xanthine oxidase and its inhibitor in hypoxia: Reoxygenation injury. Pediatrics 1996; 98: 103-107.

34. Downey JM, Miura T, Eddy LJ, Chambers DE, Mellert T, Hearse DJ, et al. Xanthine oxidase is not a source of free radicals in the ischemic rabbit heart. J Mol Cell Cardiol 1987; 19: 1053-1060.
35. Myers CL, Weiss SJ, Kirsh MM, Shepard BM, Shlafer M. Effects of supplementing hypothermic crystalloid cardioplegic solution with catalase, superoxide dismutase, allopurinol, or deferoxamine on functional recovery of globally ischemic and reperfused isolated hearts. J Thorac Cardiovasc Surg 1986; 91: 281-289.

36. de Jong JW, Schoemaker RG, de Jonge R, Bernocchi P, Keijzer E, Harrison R, et al. Enhanced expression and activity of xanthine oxidoreductase in the failing heart. J Mol Cell Cardiol 2000; 32: 2083-2089.

37. Saavedra W, Paolocci N, St John M, Skaf M, Stewart G, Xie J, et al. Imbalance between xanthine oxidase and nitric oxide synthase signaling pathways underlies mechanoenergetic uncoupling in the failing heart. Circ Res 2002; 90: 297-304.

38. White CR, Darley-Usmar V, Berrington WR, McAdams M, Gore JZ, Thompson JA, et al. Circulating plasma xanthine oxidase contributes to vascular dysfunction in hypercholesterolemic rabbits. Proc Natl Acad Sci USA 1996; 93: 8745-8749. 